\title{
Using Network Pharmacology to Systematically Decipher the Potential Mechanisms of Jisuikang in the Treatment of Spinal Cord Injury
}

\author{
Shaoshuo Li $\mathbb{D}$, ${ }^{1,2}$ Yang Shao $\mathbb{D}^{2},{ }^{2}$ Hao Chen $\left(\mathbb{D},{ }^{1,2}\right.$ and Jianwei Wang $\mathbb{D}^{1,2}$ \\ ${ }^{1}$ Nanjing University of Chinese Medicine, Nanjing 210023, China \\ ${ }^{2}$ Department of Traumatology and Orthopedics, Wuxi Affiliated Hospital of Nanjing University of Chinese Medicine, \\ Wuxi 214071, China \\ Correspondence should be addressed to Jianwei Wang; wxwangjianwei1963@126.com
}

Received 27 August 2021; Accepted 4 February 2022; Published 27 February 2022

Academic Editor: Weidong Pan

Copyright (C) 2022 Shaoshuo Li et al. This is an open access article distributed under the Creative Commons Attribution License, which permits unrestricted use, distribution, and reproduction in any medium, provided the original work is properly cited.

Objective. To identify the potential pharmacological targets of Jisuikang (JSK) for the treatment of spinal cord injury (SCI) using network pharmacology. Methods. The bioactive compounds of JSK herbs and their corresponding potential SCI targets were obtained from three traditional Chinese medicine (TCM) databases. SCI-related therapeutic target genes were obtained from the Comparative Toxicogenomics Database and the GeneCards Database. The common target genes between the JSK compounds and SCI-related therapeutic targets were screened using GO/KEGG functional enrichment and proteinprotein interaction (PPI) analyses to identify hub genes and their categories of biological function. Gene expression distribution and receiver operating characteristic curve (ROC) analyses were used to identify probable SCI-related target genes. Molecular docking was used to quantify molecular interactions between target genes and the bioactive compounds of JSK. Results. A total of 183 JSK bioactive compounds and 197 target genes for the treatment of SCI were screened and assessed. The target genes were enriched primarily in drug metabolism and in inflammation-related biological processes. Ten genes with statistical significance were identified as therapeutic SCI-related target genes of JSK. Molecular docking experiments demonstrated that the proteins of these 10 genes docked with binding energies of less than $-5 \mathrm{kcal} / \mathrm{mol}$ with the bioactive compounds in JSK. Conclusion. This study showed that the anti-SCI effects of JSK may be mediated through numerous bioactive components, multiple gene targets, and inflammation-related pathways and provided potential novel targets for directed therapies for treating SCI. These results provide a foundation for further experimental investigations into treatment options for SCI.

\section{Introduction}

Spinal cord injury (SCI) can result in immediate and devastating impacts on motor function and other essential physiological functions [1]. The incidence of SCI in the United States is approximately 50 per million people per year, with over 17000 new cases reported every year [2]. The World Health Organization (WHO) estimates that between 250000 and 500000 people suffer from SCI each year [3]. SCI patients who survive the initial injury usually suffer multisystem trauma concomitant with SCI, contributing to significant risks of associated complications [4]. It is reported that the lifetime cost of treating SCI in the US ranges between $\$ 1.1$ million and $\$ 4.7$ million, and the total cost of caring SCI patients in the US exceeds $\$ 7$ billion per year [2]. Although extensively studied and the understanding of their underlying pathophysiology has improved, effective clinical treatments for SCI are limited [5]. Because of this, complementary and alternative therapies have been increasingly used for treating SCI, especially traditional Chinese medicines (TCMs) [6]. TCM has been developing for over 2000 years and has a comprehensive theoretical system for disease diagnosis and prognosis [7]. TCM provides important guidelines for the treatment of numerous complicated diseases [7]. 
Jisuikang (JSK) is a clinical Chinese herbal formula developed using herbs described in TCM, which has shown satisfactory efficacy in several clinical trials comparing conventional SCI treatments [8-10]. JSK could significantly improve motor function and grades of spinal injury in patients after SCI, promoting the recovery and regeneration of nerve tissues. Wang et al. found that JSK promotes nerve cell regeneration by upregulating the expression of Nogo-A that reduces the growth of axotomized neurons after SCI $[11,12]$. JSK was shown to increase neurotrophic factor expression, which promotes the recovery of neurological function in SCI rats [13]. Wu et al. showed that JSK improves motor function in SCI rats by inhibiting the NgR/RhoA/ ROCK signaling pathway [14], indicating the potential of JSK to regenerate nerves. However, JSK is composed of a variety of Chinese herbs [8], making it a challenge untangling the underlying molecular mechanisms of JSK in SCI recovery.

TCM therapy takes advantage of multiple herbal components, protein targets, and biological pathways, as well as their synergism, which complicates the identification of bioactive compounds, their biological targets, and their biological mechanisms [15]. Network pharmacology reveals the potential and complex interactions between compounds in Chinese herbs and targeted diseases by systematically investigating the "drug-gene-protein-disease" interaction network [16-18]. Network pharmacology integrates system biology, pharmacology, and computational biology and provides a systematic approach to studying how TCM regulates human biological networks [19]. Network pharmacology has discovered the bioactive compounds in Yinqiao and Xianlinggubao preparations that interact with candidate proteins and biological pathways for COVID-19 [20] and osteoporosis [21], respectively.

In the present study, network pharmacology was used to screen and discover the bioactive compounds and the potential pharmacological mechanisms of JSK for the treatment of SCI. Gene expression analysis and molecular docking methods were used to verify the results of the network pharmacology analysis.

\section{Materials and Methods}

2.1. Screening Bioactive Compounds and Targets of JSK. The workflow of this study is displayed in Figure 1. The formula of JSK consists of 16 Chinese herbs: Cheqianzi (Plantaginis Semen), Chishao (Radix Paeoniae Rubra), Chuanxiong (Chuanxiong Rhizoma), Dahuang (Radix Rhei Et Rhizome), Danggui (Angelicae Sinensis Radix), Danshen (Radix Salviae), Fuling (Poria Cocos (Schw.) Wolf.), Houpo (Magnolia Officinalis Rehd Et Wils.), Huangqi (Hedysarum Multijugum Maxim.), Roucongrong (Cistanches Herba), Tubiechong (Eupolyphaga Seu Steleophaga), Wugong (Scolopendra Subspinipes Mutilans), Yinyanghuo (Epimrdii Herba), Yizhi (Alpiniae Oxyphyliae Fructus), Zexie (Alisma Orientale (Sam.) Juz.), and Zhishi (Aurantii Fructus Immaturus). The herbal compounds were found in the Traditional Chinese Medicine Systems Pharmacology Database and Analysis Platform (TCMSP, https://old.tcmsp-e. com/tcmsp.php) [22], the Bioinformatics Analysis Tool for Molecular Mechanism of Traditional Chinese Medicine (BATMAN-TCM, http://bionet.ncpsb.org.cn/batman-tcm/) [23], and the Integrative Pharmacology-Based Research Platform of Traditional Chinese Medicine (TCMIP, http:// www.tcmip.cn/TCMIP/index.php/Home/) [24]. The values for oral bioavailability (OB) and drug-likeness (DL) were set to $>30 \%$ and $>0.18$ for screening candidate bioactive compounds [25]. All the bioactive compounds were annotated using the MOL IDs from TCMSP. Target genes of the bioactive compounds were obtained from TCMSP, and their official gene names were obtained from the UniProt Knowledgebase (UniProtKB, https://www.uniprot.org/).

2.2. Predicting Target Genes of SCI. The Human Gene Database (GeneCards, https://www.genecards.org/) [26] and the Comparative Toxicogenomics Database (CTD, http://ctdbase. org/) [27] were used to find potential therapeutic target genes for SCI using "spinal cord injury" as the search term.

2.3. Constructing the Interaction Network of JSK and Common Target Genes. Common target genes between JSK and SCI were identified using the VennDiagram package [28] in R 3.6.2 (https://www.r-project.org/). The interaction relationships between JSK bioactive compounds and the common target genes were determined, and an interaction network was constructed using Cytoscape software 3.8.2 (https://cytoscape.org/) [29].

\subsection{Functional Enrichment Analysis. Gene Ontology (GO)} enrichment analysis and the Kyoto Encyclopedia of Genes and Genomes (KEGG) signaling pathway enrichment analysis were performed to identify possible biological functions of the common target genes using the Database for Annotation, Visualization and Integrated Discovery 6.8 (DAVID 6.8, https://david.ncifcrf.gov/) [30]. The categories of cellular component, molecular function, and biological process were included in the GO terms. A false discovery rate $(\mathrm{FDR})<0.05$ was used to indicate significant differences among GO terms and KEGG pathways.

2.5. Protein-Protein Interaction Analysis and Identification of Hub Genes. The Search Tool for the Retrieval of Interacting Genes (STRING V11, https://www.string-db.org/) [31] was used to construct a protein-protein interaction (PPI) network of the common target genes. The minimum required interaction score was set to 0.400 . The PPI network was developed using Cytoscape. The CytoNCA plugin for Cytoscape [32] was used to perform a topological network analysis that included betweenness centrality (BC), closeness centrality (CC), and degree centrality (DC) to identify hub genes among the common target genes.

2.6. Data Validation of Hub Genes. Gene expression distribution and receiver operating characteristic (ROC) curves of the hub genes were generated and used to investigate the 


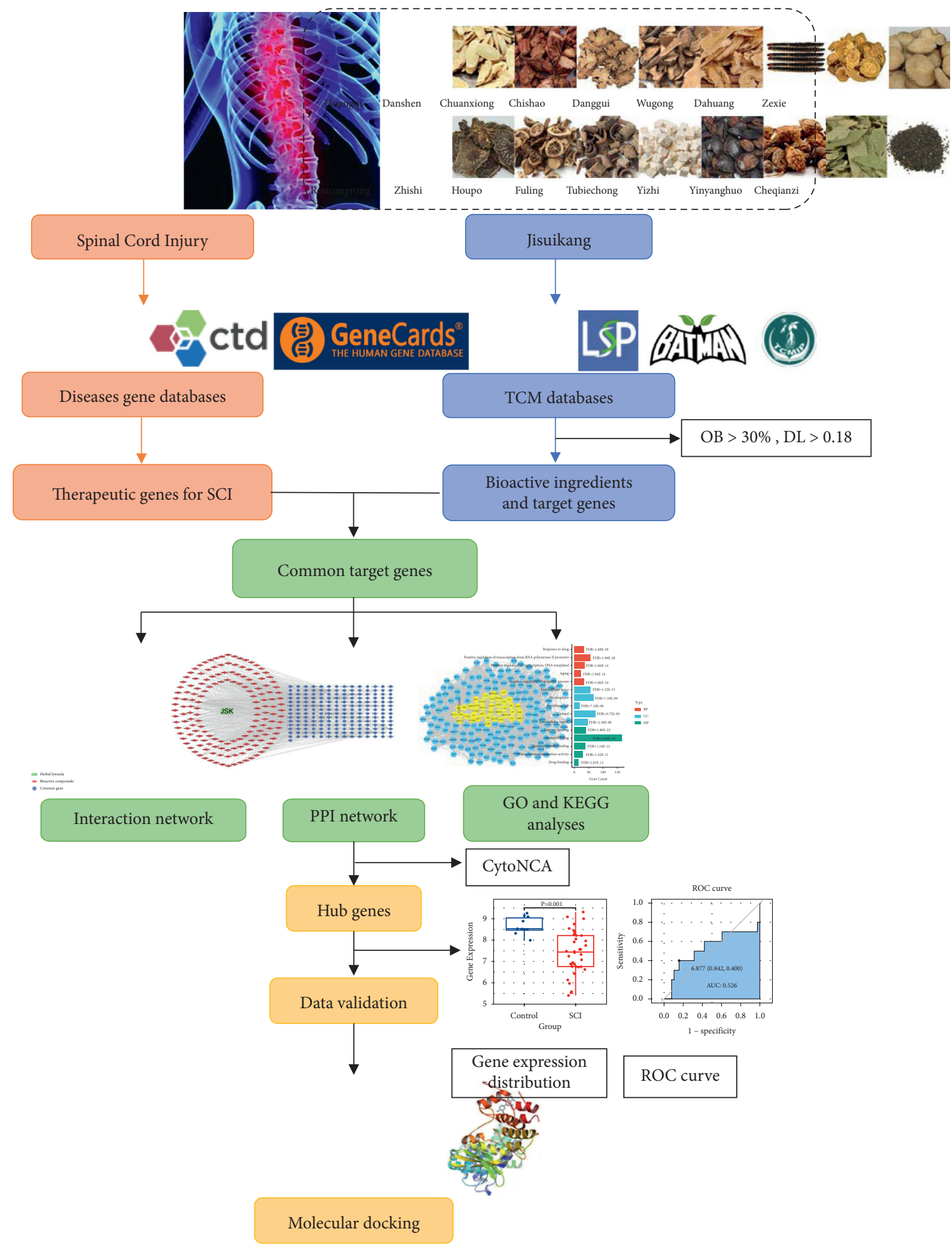

FIGURE 1: Workflow for the present study.

association between the hub genes and prognoses in patients with SCI. The gene microarray dataset GSE151371 was downloaded from the Gene Expression Omnibus database (GEO, http://www.ncbi.nlm.nih.gov/geo/) and provided the global gene expression in peripheral white blood cells from 10 healthy individuals without a history of SCI and 38 individuals who suffered traumatic SCI. The gene expression distributions and ROC curves of the identified hub genes between healthy individuals and the SCI individuals were assessed in these two groups to determine their probability as targets for treating SCI. Hub genes that displayed statistically significant differences (a BH-adjusted $p<0.05)$ and areas under the curve (AUC) $>0.7$ in the ROC analysis were considered key genes relevant to possible treatments for SCI. 
2.7. Molecular Docking of Bioactive JSK Compounds and Proteins Encoded by Key Genes. The structure files (PDB files) of the protein products of key genes identified through gene expression distribution and ROC analyses were obtained from the Protein Data Bank (PDB, https://www.rcsb.org/) [33]. AutoDockTools V1.5.6 (http://autodock.scripps.edu/) was used to remove excess ligands, water molecules, and protein chains from the protein structures and to add hydrogens to the proteins. Bioactive compounds of JSK that were correlated with proteins encoded by the hub genes were used as molecular ligands in the docking program. The molecular structure files of bioactive JSK compounds were acquired from the PubChem database (https://pubchem.ncbi.nlm.nih.gov/), and Chem3D (https://www.codeweavers.com/) was used to construct the 3D models of the molecules (mol2 files). The PDB files of the target proteins and mol2 files of the bioactive compounds were converted into "PDBQT" formats using AutoDockTools. The docking coordinates were identified using the grid boxes in AutoDockTools. AutoDock Vina (http://vina.scripps.edu/) [34] was used to dock the molecular ligands to the corresponding proteins. The results of molecular docking experiments were analyzed and visualized using Discovery Studio 2016 and PyMOL (https://pymol.org/).

\section{Results}

3.1. Bioactive Compounds and Target Genes. The number of bioactive compounds found in the herbs comprising JSK is shown in Table 1. After removing duplicate compounds, a total of 183 JSK bioactive compounds were identified. There were 233 potential target genes of these bioactive compounds identified. In addition, the search of the CTD and GeneCards databases identified 16184 and 6348 SCI-related target genes, respectively.

\subsection{Construction of "JSK-Bioactive Compounds-Common} Target Genes" Interaction Network. There were 197 genes in common between JSK compound target genes and SCIrelated genes (Figure 2). An interaction network analysis identified 359 nodes (1 herbal formula, 161 bioactive compounds, and 197 shared target genes) and 1632 edges (Figure 3). According to the gene count, the top 15 bioactive compounds in the network are listed in Table 2.

3.3. GO and KEGG Enrichment Analyses. The GO terms most enriched in each category correlated to the bioactive compounds in JSK are listed in Figure 4. The top 5 enriched GO terms were enzyme binding, positive regulation of transcription from RNA polymerase II promoter, response to drug, protein binding, and extracellular space. KEGG signaling pathway analysis revealed that most of the common target genes have associations with cancer and inflammation-related pathways (Table 3).

3.4. PPI Analysis and Identification of Hub Genes. The PPI network of common genes consisted of 197 nodes and 3829 edges. According to topological network analysis,
TABLE 1: The number of bioactive compounds in herbs from JSK.

\begin{tabular}{lcccc}
\hline Chinese herbs in JSK & TCMSP & $\begin{array}{c}\text { BATMAN- } \\
\text { TCM }\end{array}$ & TCMIP & Total \\
\hline Cheqianzi & 7 & 1 & 25 & 11 \\
Chishao & 13 & 9 & 16 & 16 \\
Chuanxiong & 6 & 91 & 34 & 11 \\
Dahuang & 7 & 30 & 79 & 13 \\
Danggui & 2 & 83 & 37 & 5 \\
Danshen & 58 & 39 & 34 & 62 \\
Fuling & 6 & 18 & 32 & 15 \\
Houpo & 2 & 42 & 32 & 6 \\
Huangqi & 16 & 24 & 24 & 21 \\
Roucongrong & 6 & 18 & 32 & 7 \\
Tubiechong & - & 1 & - & 1 \\
Wugong & - & 5 & - & 1 \\
Yinyanghuo & 21 & 9 & 17 & 22 \\
Yizhi & 3 & 19 & 20 & 4 \\
Zexie & 7 & 14 & 21 & 9 \\
Zhishi & 17 & 12 & 27 & 22 \\
\hline
\end{tabular}

JSK: Jisuikang; TCMSP: Traditional Chinese Medicine Systems Pharmacology Database and Analysis Platform; BATMAN-TCM: Bioinformatics Analysis Tool for Molecular Mechanism of Traditional Chinese Medicine; TCMIP: Integrative Pharmacology-Based Research Platform of Traditional Chinese Medicine.

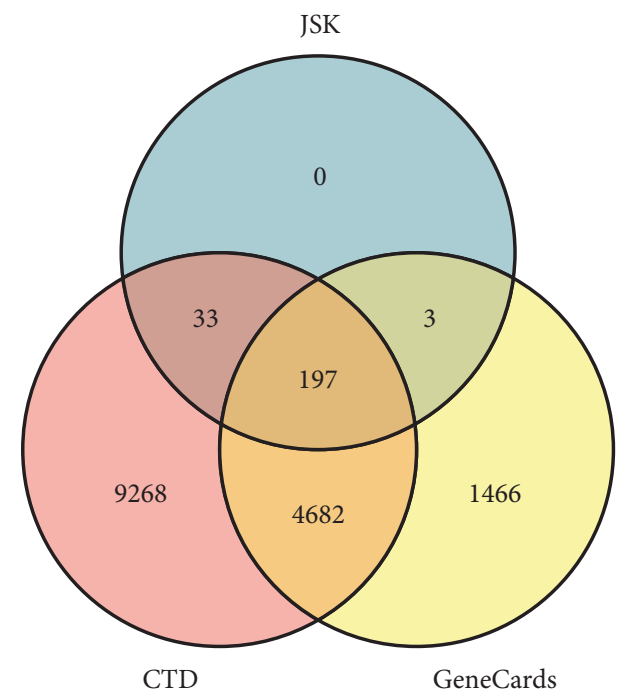

FIGURE 2: Venn diagram for JSK-related target genes and SCIrelated target genes.

PPI nodes are considered significant targets if the DC values were greater than twofold the median DC [35]. The median DC was set to a threshold $>62$ to identify significant nodes and generate a subnetwork. In the subnetwork, nodes where both $\mathrm{BC}$ and $\mathrm{CC}$ values were greater than their median $(\mathrm{BC}>2.34, \mathrm{CC}>0.95)$ were further extracted as hub genes, and a new core network composed of hub genes was constructed (Figure 5). Important therapeutic target genes of JSK in the treatment of SCI were identified as $A K T 1, C A S P 3, C C N D 1$, CXCL8, EGF, EGFR, FOS, IL6, JUN, MAPK1, MAPK3, MAPK8, MMP2, MMP9, MYC, PTGS2, STAT3, TP53, and VEGFA. 


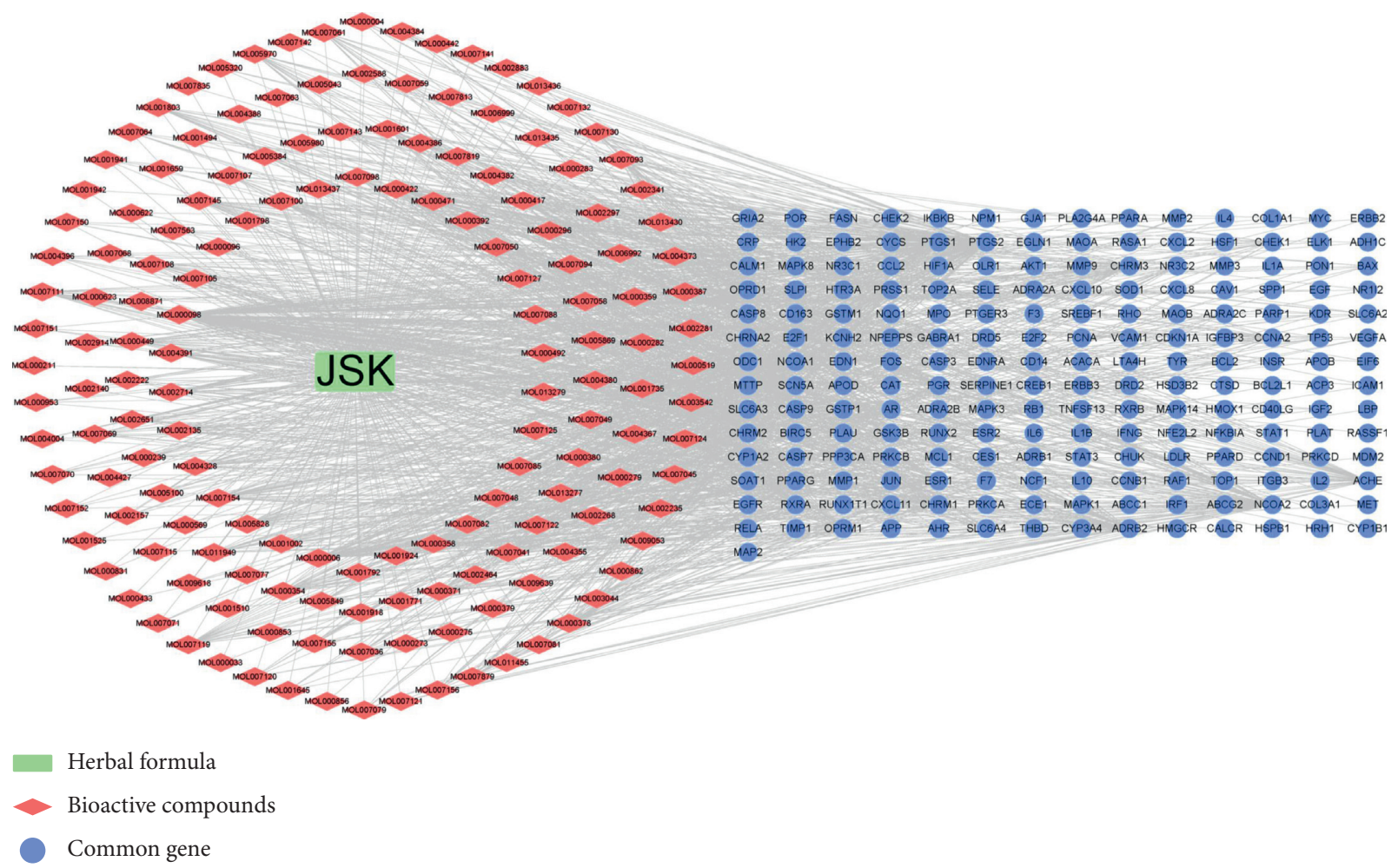

FIGURE 3: JSK-bioactive compounds-common target genes interaction network. The green rectangle represents JSK; the red diamonds represent the bioactive compounds of JSK; the blue circles represent the target genes in common between JSK and SCI.

TABLE 2: The top 15 bioactive compounds in JSK.

\begin{tabular}{|c|c|c|c|c|c|}
\hline MOL ID & Bioactive compounds & $\begin{array}{l}\text { Gene } \\
\text { count }\end{array}$ & $\begin{array}{l}\text { OB } \\
(\%)\end{array}$ & $\mathrm{DL}$ & Corresponding herbs \\
\hline MOL000098 & Quercetin & 119 & 46.43 & 0.28 & $\begin{array}{l}\text { Cheqianzi, Huangqi, Roucongrong, } \\
\text { Yinyanghuo }\end{array}$ \\
\hline MOL000006 & Luteolin & 46 & 36.16 & 0.25 & Danshen, Yinyanghuo, Zhishi \\
\hline MOL000422 & Kaempferol & 40 & 41.88 & 0.24 & Dahuang, Huangqi, Yinyanghuo \\
\hline MOL007154 & Tanshinone IIA & 31 & 49.89 & 0.40 & Danshen \\
\hline MOL000378 & 7-O-methylisomucronulatol & 27 & 74.69 & 0.30 & Huangqi \\
\hline MOL005828 & Nobiletin & 27 & 61.67 & 0.52 & Yizhi \\
\hline MOL002714 & Baicalein & 24 & 33.52 & 0.21 & Cheqianzi, Chishao \\
\hline MOL004373 & Anhydroicaritin & 24 & 45.41 & 0.44 & Yinyanghuo \\
\hline MOL000358 & Beta-sitosterol & 23 & 36.91 & 0.75 & $\begin{array}{l}\text { Dahung, Danggui, Houpo, Huangqi, } \\
\text { Roucongrong, }\end{array}$ \\
\hline MOL000392 & Formononetin & 23 & 69.67 & 0.21 & Huangqi \\
\hline MOL004328 & Naringenin & 23 & 59.29 & 0.21 & Yizhi \\
\hline MOL004380 & $\begin{array}{l}\text { C-Homoerythrinan, 1,6-didehydro-3,15,16- } \\
\text { trimethoxy-, (3.beta.)- }\end{array}$ & 23 & 39.14 & 0.49 & Yinyanghuo \\
\hline MOL007119 & Miltionone I & 23 & 49.68 & 0.32 & Danshen \\
\hline MOL000449 & Stigmasterol & 22 & 43.83 & 0.76 & $\begin{array}{c}\text { Danggui, Danshen, Cheqianzi, Chishao, } \\
\text { Yizhi }\end{array}$ \\
\hline MOL000354 & Isorhamnetin & 22 & 49.60 & 0.31 & Huangqi \\
\hline
\end{tabular}

OB: oral bioavailability; DL: drug-likeness.

3.5. Hub Gene Validation. The results of the gene expression distribution of hub genes in the GSE151371 dataset (except EGFR, IL6, and MMP2, which were not in the GSE151371 dataset) show that 10 hub genes were downregulated in patients with SCI (Figure 6). Expression levels of JUN, MAPK1, MAPK3, $M M P 9, S T A T 3$, and VEGFA were higher in the SCI group than in the control group. ROC curves and associated AUC values of these hub genes are presented in Figure 7. After combining the results of gene expression distributions and ROC curves, 10 key genes were found to have significant expression changes in SCI compared to the control: CCND1, CXCL8, FOS, JUN, MAPK3, MMP9, MYC, PTGS2, TP53, and VEGFA. 


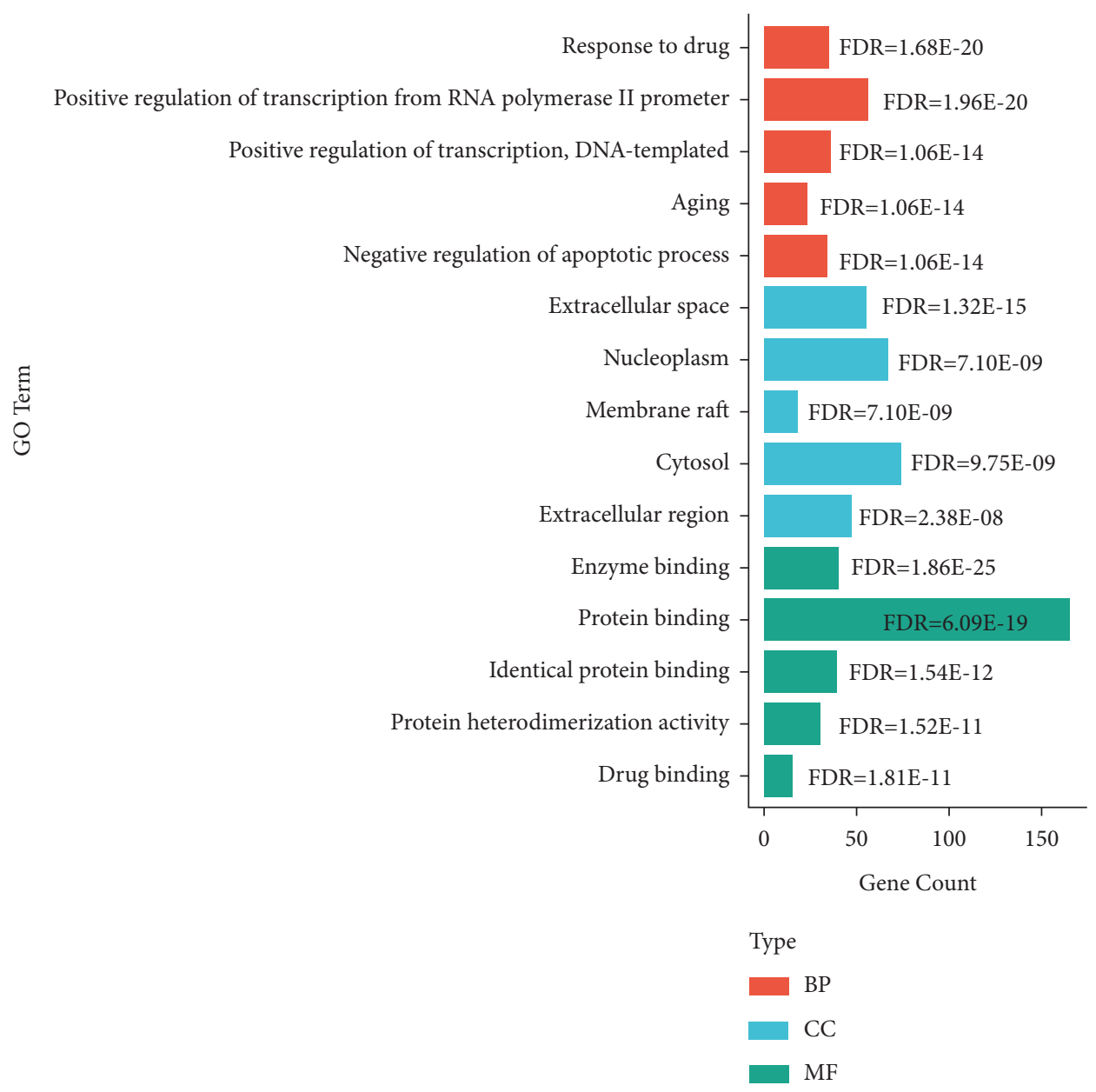

FIgURE 4: The bar plot of top five enriched GO terms of common target genes. BP: biological process; CC: cellular component; MF: molecular function; FDR: false discovery rate.

TABLE 3: Significantly enriched KEGG signaling pathways.

\begin{tabular}{lccr}
\hline Pathway ID & Pathway term & Gene count & FDR \\
\hline Hsa04066 & HIF-1 signaling pathway & 23 & 35 \\
Hsa04151 & PI3K-Akt signaling pathway & 21 & $6.78 E-11$ \\
Hsa04620 & Toll-like receptor signaling pathway & 27 & $2.06 E-11$ \\
Hsa04668 & TNF signaling pathway & 17 & $2.14 E-17$ \\
Hsa05133 & Pertussis & 20 & $2.66 E-10$ \\
Hsa05142 & Chagas disease (American trypanosomiasis) & 21 & $9.23 E-11$ \\
Hsa05145 & Toxoplasmosis & 25 & $3.54 E-11$ \\
Hsa05152 & Tuberculosis & 22 & $1.19 E-10$ \\
Hsa05160 & Hepatitis C & 36 & $1.19 E-10$ \\
Hsa05161 & Hepatitis B & 25 & $2.60 E-23$ \\
Hsa05164 & Influenza A & 55 & $9.23 E-11$ \\
Hsa05200 & Pathways in cancer & 29 & $8.75 E-24$ \\
Hsa05205 & Proteoglycans in cancer & 17 & $1.63 E-12$ \\
Hsa05210 & Colorectal cancer & 21 & $2.08 E-11$ \\
Hsa05212 & Pancreatic cancer & 23 & $1.43 E-15$ \\
Hsa05215 & Prostate cancer & 20 & $4.22 E-15$ \\
Hsa05219 & Bladder cancer & 17 & $1.51 E-18$ \\
Hsa05220 & Chronic myeloid leukemia & 18 & $1.52 E-10$ \\
Hsa05222 & Small cell lung cancer & 18 & $2.03 E-10$ \\
Hsa05223 & Non-small cell lung cancer & & $2.85 E-13$ \\
\hline
\end{tabular}

FDR: false discovery rate. 


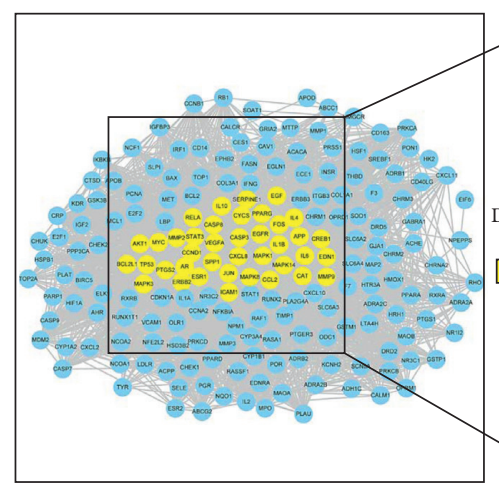

196 nodes and 3829 edges

(a)

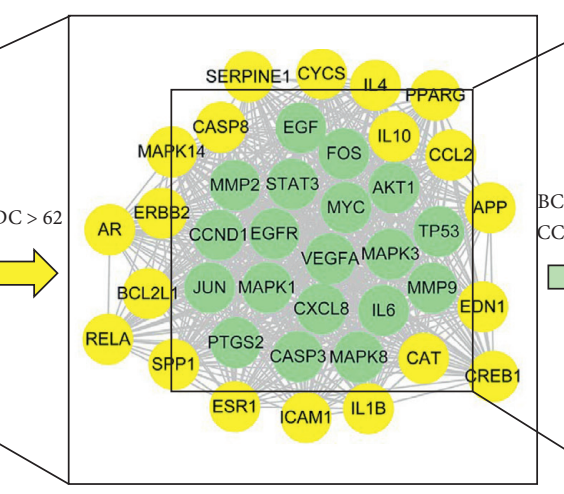

39 nodes and 696 edges

(b)

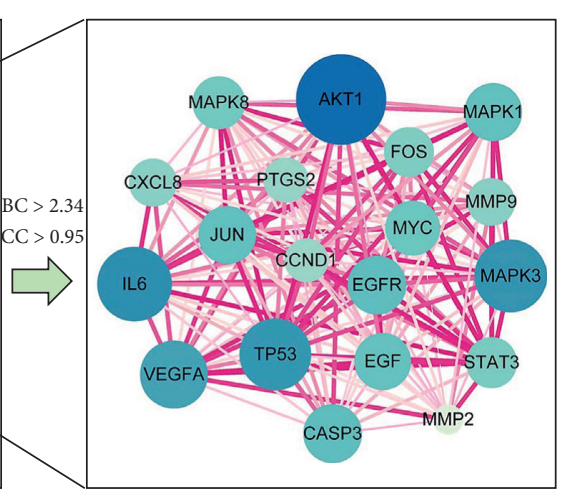

19 nodes and 171 edges

(c)

Figure 5: The screening process of hub genes in the PPI network.

3.6. Results of Molecular Docking. The protein products of the 10 key genes and the corresponding JSK compounds were chosen for molecular docking to assess their interactions. The affinity binding energies between the target proteins and the bioactive compounds were calculated (Table 4). The efficacy of molecular docking is considered to be good if the affinity binding energy is $<-5.0 \mathrm{kcal} / \mathrm{mol}$. The docking results show that quercetin, luteolin, tanshinone IIA, and naringenin had strong binding energies with their corresponding SCI proteins. The molecular dockings of the best combination of key gene proteins and their corresponding JSK compounds are presented in Figure 8.

\section{Discussion}

Individuals might face decades with permanent SCI-related disabilities. Advances in understanding the pathological mechanism of SCI have improved clinical management strategies for treating SCI, decreasing morbidity, and improving functional outcomes in patients with SCI [1]. However, randomized clinical trials have not demonstrated the efficacy and safety of conventional reparatory therapies for the recovery of motor function in SCI patients. TCM therapies, including JSK, Buyang Huanwu decoction, and Governor Vessel electroacupuncture, have provided varying degrees of therapeutic results for SCI $[8,36,37]$.

The TCM theory describes the pathogenesis of SCI as a "deficiency of kidney governor, stasis of governor pulse, and dereliction of duty of cardinal command." JSK was developed to investigate its effects in the treatment of SCI. According to TCM, the herbal compounds of JSK have the following therapeutic effects: Huangqi tonifies Qi and nourishes blood; Cheqianzi, Fuling, and Zexie help invigorate the spleen to treat diuresis; Roucongrong, Yinyanghuo, and Yizhi warm the kidney and strengthen Yang; Houpo and Zhishi regulate Qi to relieve pain; Chishao, Chuanxiong, Dahuang, Danggui, Danshen, Tubiechong, and Wugong activate blood circulation, ameliorating stasis and dredging meridians. Clinical studies have shown that JSK treatment provides significant improvement in functional recovery and the quality of life of SCI patients. However, the complex herbal composition and synergistic effects of JSK complicate the discovery of the specific mechanisms of JSK compounds for the treatment of SCI. We performed network pharmacology analysis to discover the potential bioactive compounds, biological targets, and molecular processes of JSK therapy for SCI.

4.1. Main Findings. The bioactive compounds in JSK are quercetin, luteolin, kaempferol, tanshinone IIA, nobiletin, baicalein, 7-O-methylisomucronulatol, anhydroicaritin, beta-sitosterol, formononetin, naringenin, C-Homoerythrinan, 1,6-didehydro-3,15,16-trimethoxy-, (3.beta.)-, miltionone I, stigmasterol, and isorhamnetin (Table 2). Quercetin protects the spinal cord and regulates secondary oxidative stress in SCI rats by inhibiting the activation of the p38MAPK/iNOS signaling pathway [38]. By activating the Nrf2/glutamate-cysteine ligase pathway, luteolin shows antioxidant, anti-inflammatory, and neuroprotective effects against SCI [39, 40]. Kaempferol reduces oxidative stress and inflammatory responses in SCI by inhibiting the MAPKs$\mathrm{NF}-\kappa \mathrm{B}$ and pyroptosis signaling pathways [41]. Tanshinone IIA improves functional recovery after SCI-induced lower urinary tract dysfunction [42]. Baicalein contributes to the functional recovery of SCI by dampening pyroptosis and alleviating endoplasmic reticulum stress-mediated apoptosis [43]. These studies support our findings of the bioactive compounds in JSK and their therapeutic role in SCI.

GO enrichment analysis revealed that the common genes were involved in regulation of transcription, enzyme binding and protein binding, response to drug, and extracellular space, indicating that the therapeutic effects of JSK may be associated with transcriptional regulation of target genes, drug metabolism, and various cell metabolic pathways. KEGG pathway analysis revealed that JSK might produce anti-SCI effects via multiple signaling pathways, including those in cancer, and the TNF, HIF-1, Toll-like receptor, and PI3K-Akt signaling pathways. The inflammatory response of nerve cells potentiates SCI damage and hinders functional recovery after SCI [44]. The PI3K-Akt pathway regulates regeneration of SCI in rats by attenuating the inflammatory response [45]. Hypoxia-inducible factor-1 (HIF-1) was found to reduce inflammation in SCI via miR-380-3p/ 


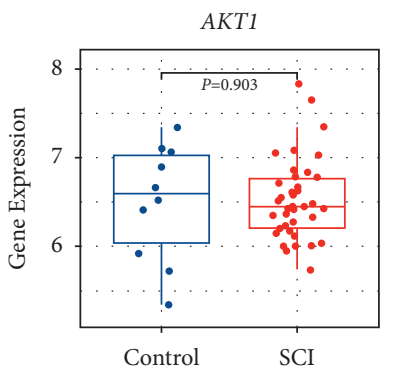

(a)

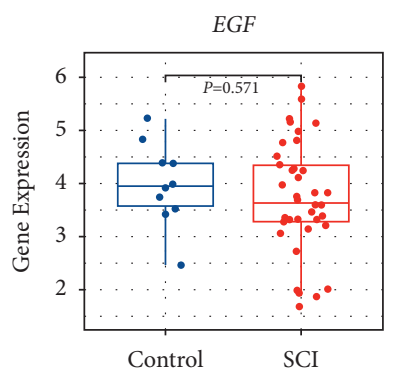

(e)

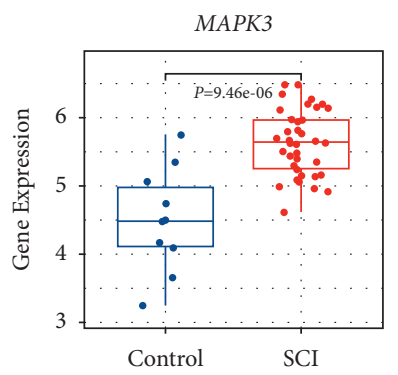

(i)

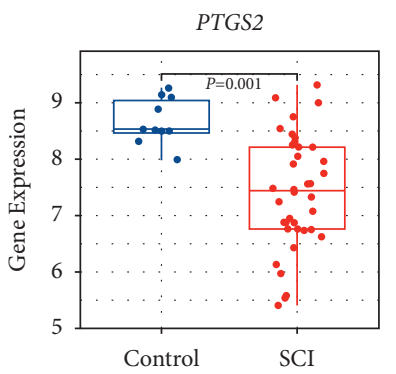

(m)

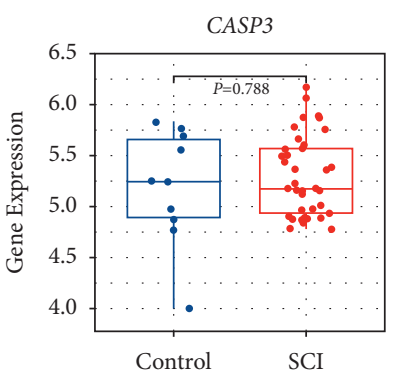

(b)

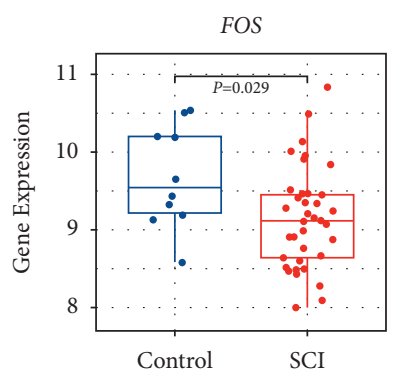

(f)

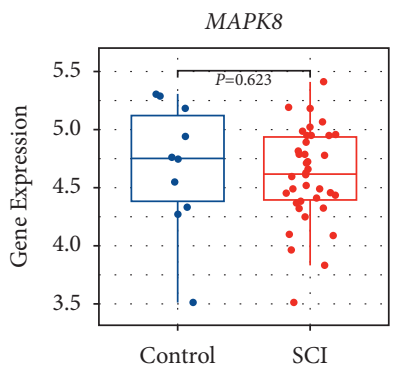

(j)

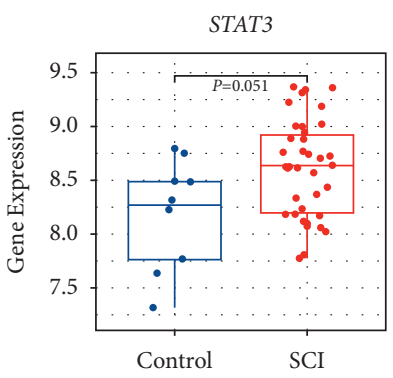

(n)

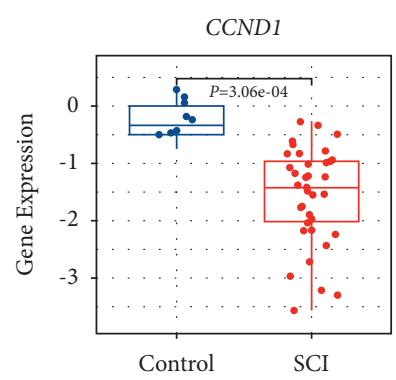

(c)

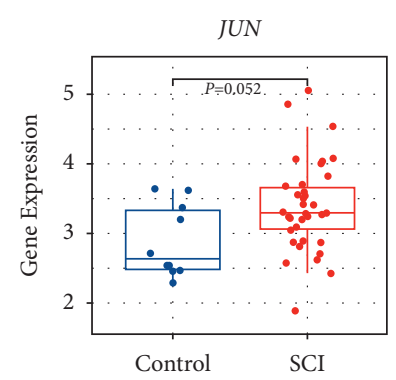

(g)

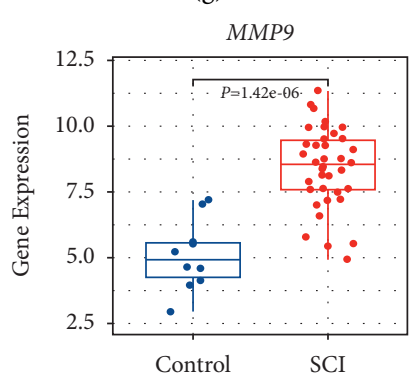

(k)

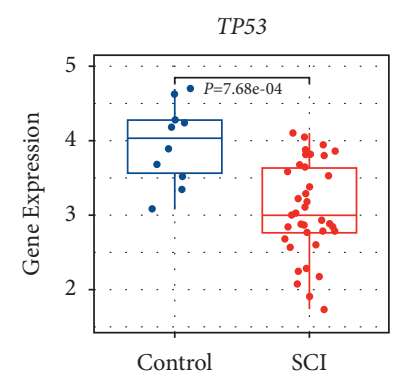

(o)

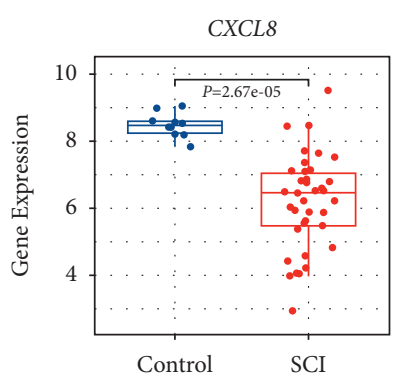

(d)

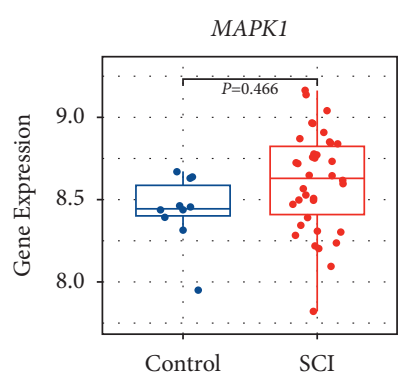

(h)

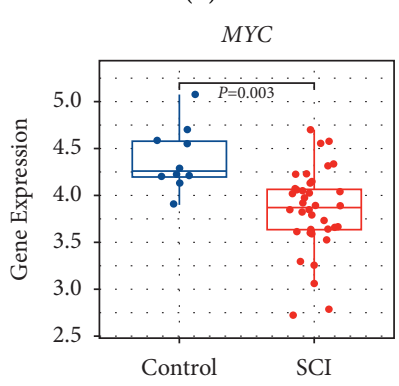

(l)

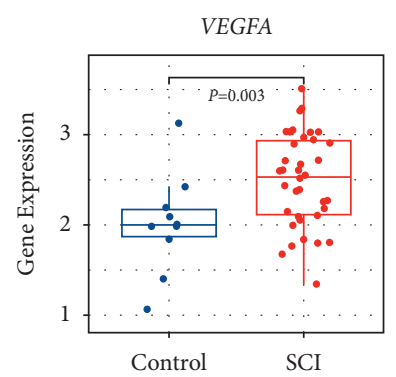

(p)

FIgUre 6: Boxplots of hub gene expression between the control group and the SCI group. (a-p) AKT1, CASP3, CCND1, CXCL8, EGF, FOS, JUN, MAPK1, MAPK3, MAPK8, MMP9, MYC, PTGS2, STAT3, TP53, and VEGFA.

NLRP3 and Circ 0001723 [46]. TNF was identified from a different network analysis as a major hub for inflammation following SCI [47]. In addition, certain drugs used to treat SCI are highly associated with TNF-related pathways. Overall, our results are consistent with these studies, indicating that JSK may exert anti-SCI effects through the above pathways.

We identified 19 proteins from hub genes using PPI network analysis and confirmed that 10 were associated with JSK compounds in the treatment of SCI (CCND1, CXCL8, FOS, JUN, MAPK3, MMP9, MYC, PTGS2, TP53, and VEGFA). The affinity values of all docking results were calculated to be less than $-5.0 \mathrm{kcal} / \mathrm{mol}$, indicating acceptable binding affinities between the 10 key proteins and the corresponding compounds in JSK. Our findings are consistent with numerous investigations linking proteins and pathways to SCI. Epigenetic silencing of CCND1 in bone marrow-derived mesenchymal stem cells provides protective effects and accelerates SCI repair in rats [48]. There is evidence indicating that inhibition of the MAPK3/MAPK1 pathway could reduce inflammation and tissue injury in mice with SCI [49]. Activation of the cannabinoid-2 receptor attenuates neurological deficits in SCI by inhibiting MMP9 expression [50]. The transcription factor p53 plays an 


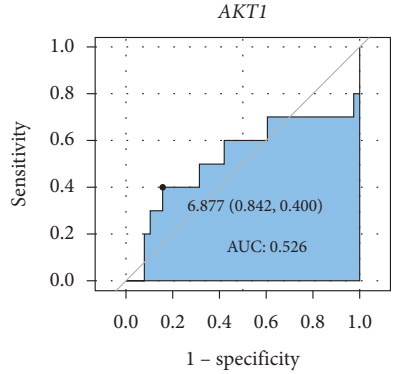

(a)

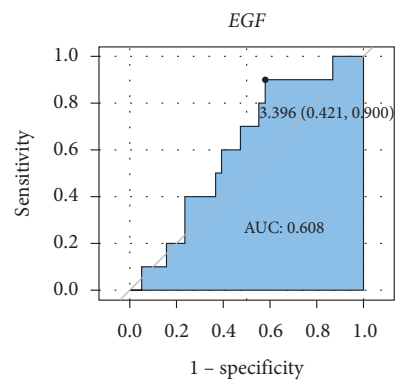

(e)

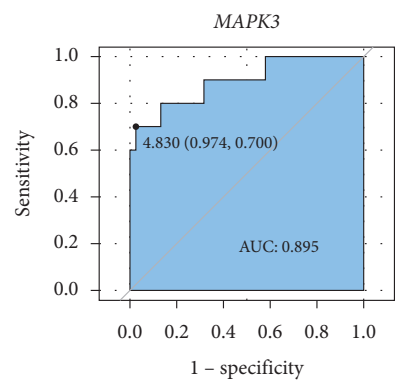

(i)

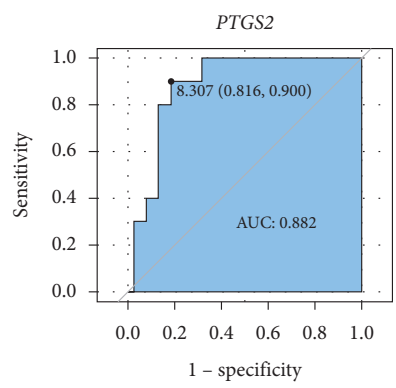

(m)

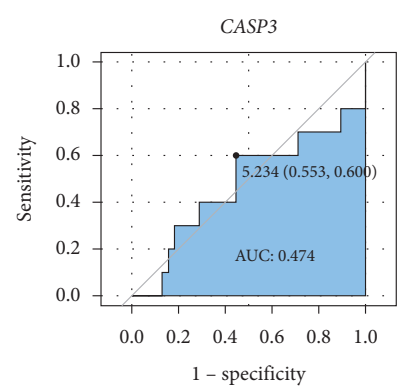

(b)

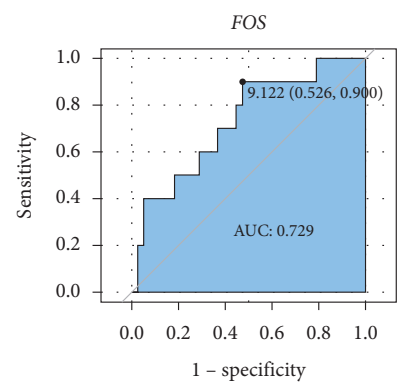

(f)

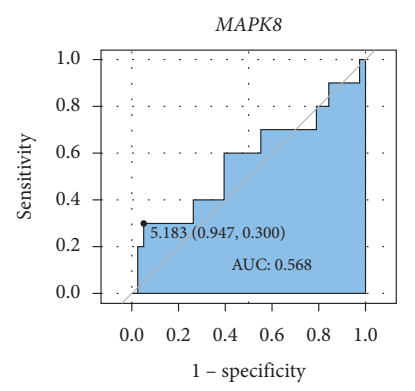

(j)

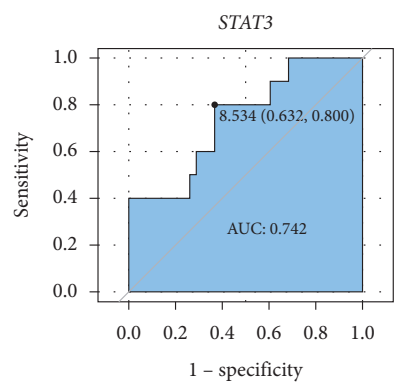

(n)

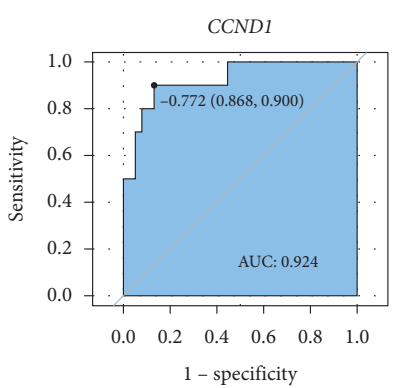

(c)

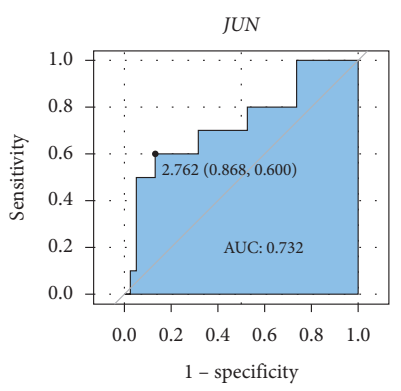

(g)

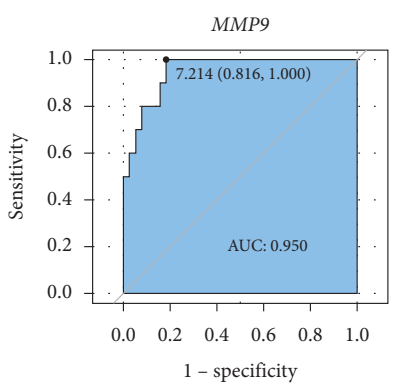

(k)

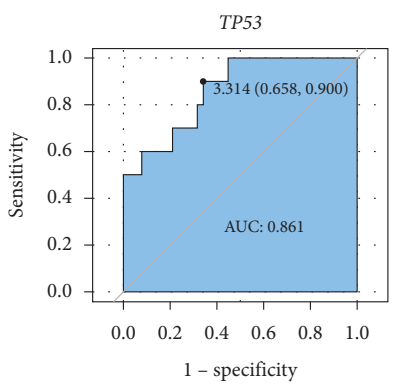

(o)

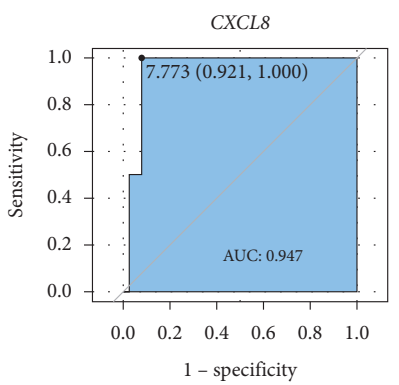

(d)

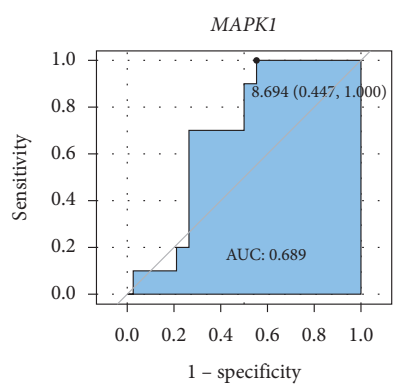

(h)

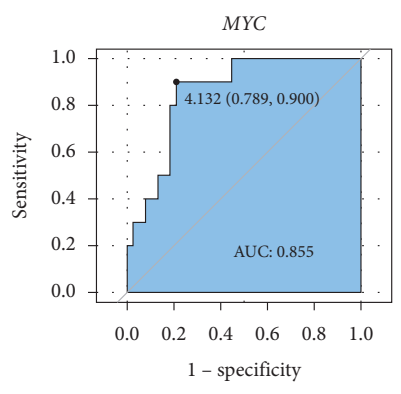

(1)

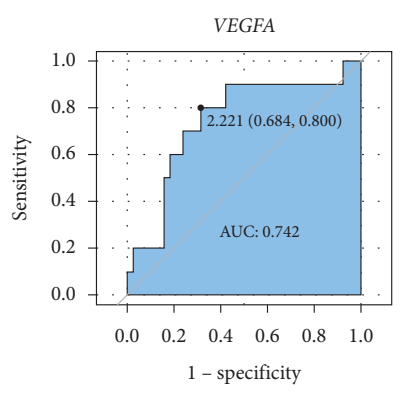

(p)

FIgURE 7: Receiver operator characteristic curves of hub genes in SCI patients. (a-p) AKT1, CASP3, CCND1, CXCL8, EGF, FOS, JUN, MAPK1, MAPK3, MAPK8, MMP9, MYC, PTGS2, STAT3, TP53, and VEGFA.

important role in regulating the regeneration and functional recovery of axons after SCI [51]. The astrocytes with dysregulated CXCL8 display restored sensitivity to inflammatory stimuli following spinal cord lesion, suggesting that CXCL8 contributes to the regulation of astrocyte-mediated inflammation [52]. MiR-152 inhibits inflammatory responses and promotes the recovery of the SCI in mice through the c-jun $\mathrm{N}$-terminal kinase pathway [53]. Inhibition of miR-17-5p upregulates the expression of VEGFA in mesenchymal stem cells (MSCs) and promotes the angiogenic ability of MSCs to repair SCI [54].
This study uncovered the multiple bioactive compounds, multiple target genes, and multiple pathways characteristics of JSK in the treatment of SCI. The above analysis results have given a general direction for research related to TCM therapy for SCI.

4.2. Limitations and Future Perspectives. There are limitations associated with the present study. Direct experimental validation of our findings is lacking, although our results are consistent with findings from other studies. In addition, all 
TABLE 4: Results of molecular docking between key proteins and bioactive compounds in JSK.

\begin{tabular}{|c|c|c|c|c|c|}
\hline $\begin{array}{l}\text { Key } \\
\text { proteins }\end{array}$ & $\begin{array}{l}\text { Core } \\
\text { compounds }\end{array}$ & $\begin{array}{l}\text { Affinity (kcal/ } \\
\text { mol) }\end{array}$ & $\begin{array}{c}\text { Key } \\
\text { proteins }\end{array}$ & Core compounds & $\begin{array}{l}\text { Affinity (kcal/ } \\
\text { mol) }\end{array}$ \\
\hline CCND1 & Luteolin & -7.2 & PTGS2 & 7-O-methylisomucronulatol & -8.7 \\
\hline CCND1 & Quercetin & -7.6 & PTGS2 & Baicalein & -9.6 \\
\hline CXCL8 & Quercetin & -7.8 & PTGS2 & Beta-sitosterol & -7.2 \\
\hline FOS & Baicalein & -5.7 & PTGS2 & $\begin{array}{c}\text { C-Homoerythrinan, 1,6-didehydro-3,15,16-trimethoxy- } \\
\text {, (3.beta.)- }\end{array}$ & -6.8 \\
\hline FOS & Quercetin & -5.7 & PTGS2 & Formononetin & -9.7 \\
\hline FOS & Tanshinone IIA & -6.7 & PTGS2 & Kaempferol & -9.5 \\
\hline JUN & Beta-sitosterol & -6.7 & PTGS2 & Luteolin & -10.1 \\
\hline JUN & Formononetin & -5.6 & PTGS2 & Miltionone I & -10.1 \\
\hline JUN & Kaempferol & -5.6 & PTGS2 & Naringenin & -9.4 \\
\hline JUN & Luteolin & -5.9 & PTGS2 & Nobiletin & -8.8 \\
\hline JUN & Nobiletin & -5.4 & PTGS2 & Quercetin & -10.2 \\
\hline JUN & Quercetin & -5.9 & PTGS2 & Stigmasterol & -9.0 \\
\hline JUN & Tanshinone IIA & -6.8 & PTGS2 & Tanshinone IIA & -9.9 \\
\hline MAPK3 & Naringenin & -8.6 & TP53 & Baicalein & -7.8 \\
\hline MMP9 & Baicalein & -10.4 & TP53 & Luteolin & -7.5 \\
\hline MMP9 & Luteolin & -10.7 & TP53 & Nobiletin & -7.1 \\
\hline MMP9 & Nobiletin & -7.1 & TP53 & Quercetin & -7.6 \\
\hline MMP9 & Quercetin & -10.7 & TP53 & Tanshinone IIA & -7.9 \\
\hline MMP9 & Tanshinone IIA & -8.2 & VEGFA & Baicalein & -7 \\
\hline MYC & Quercetin & -7.7 & VEGFA & Luteolin & -7.2 \\
\hline MYC & Tanshinone IIA & -7.7 & VEGFA & Quercetin & -6.8 \\
\hline
\end{tabular}

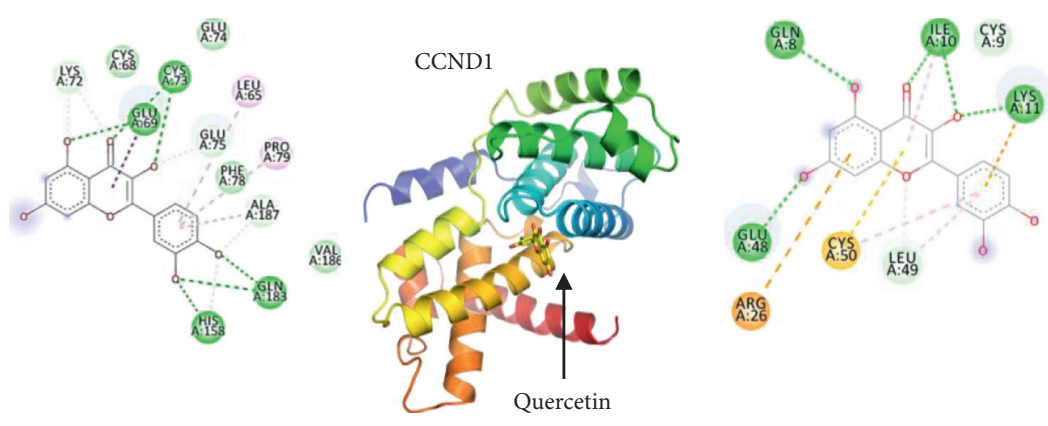

Interactions

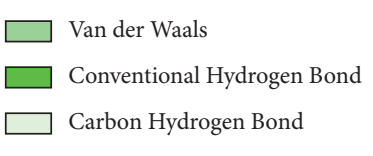

(a)
Interactions
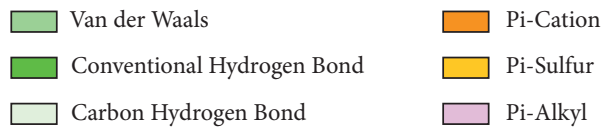

(b)

FIgURE 8: Continued.

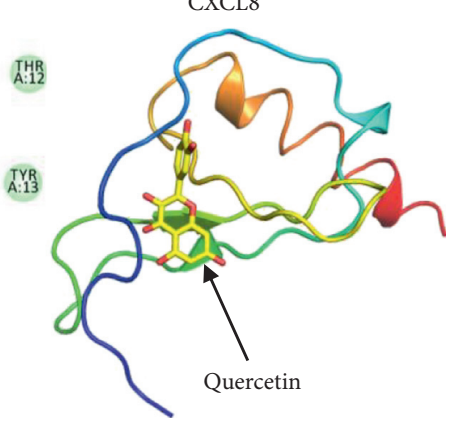


Aitip

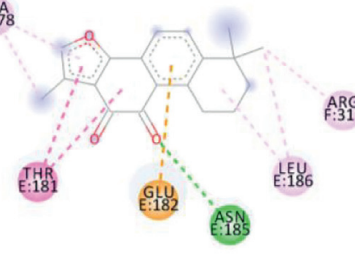

Interactions

Conventional Hydrogen Bond

$\square$ Pi-Anion

$\square$ Anide-Pi Stacked

(c)
A:25.

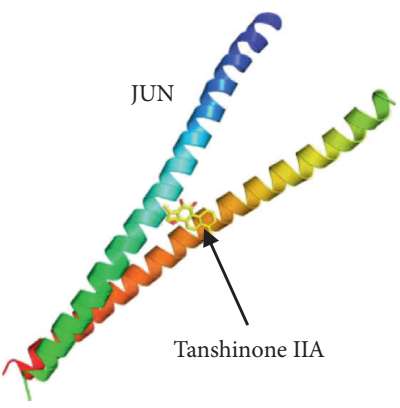

Interactions

Conventional Hydrogen Bond

Alkyl

Pi-Alkyl

(d)
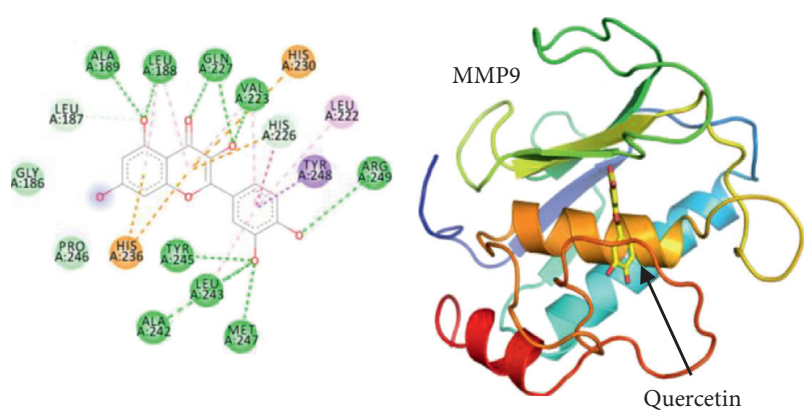

Interactions

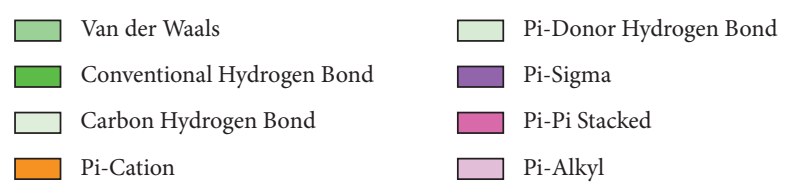

(f)

Apis

(e)
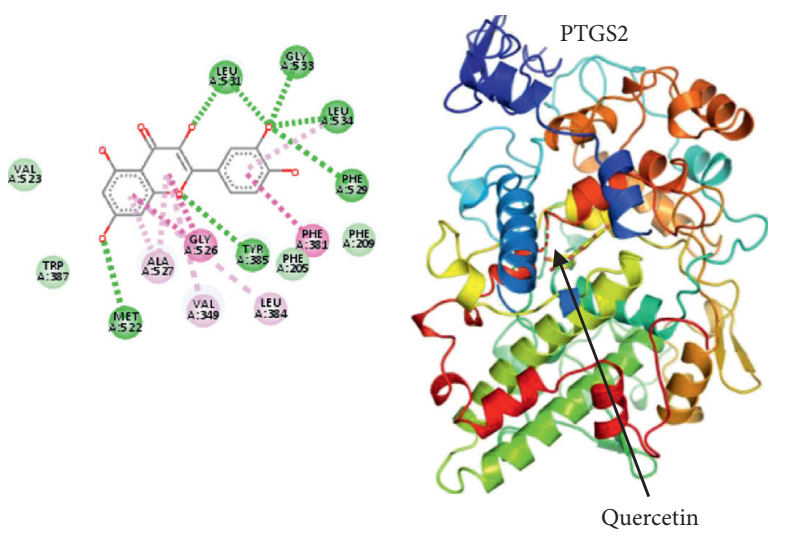

Interactions

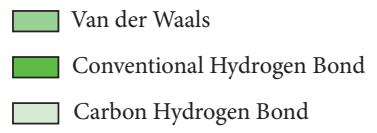

Pi-Pi T-shaped

Pi-Pi Stacked

$\square$ Pi-Alkyl

(g)

(h)

FIGURE 8: Continued. 


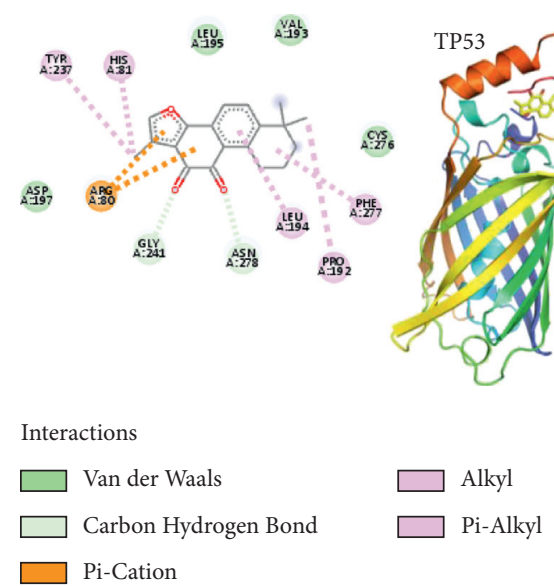

(i)
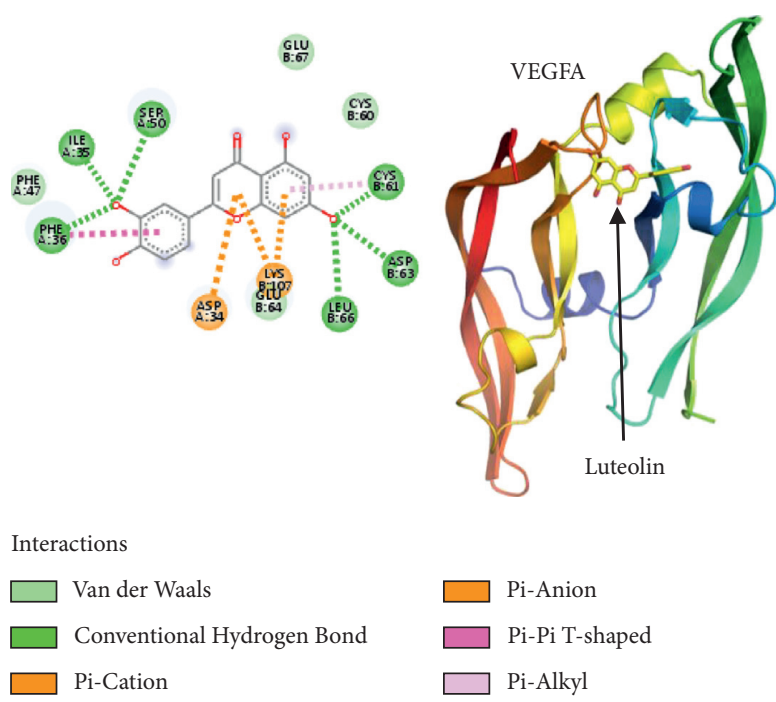

$(\mathrm{j})$

FIGURE 8: Molecular docking of protein receptors of key proteins with corresponding bioactive compounds of JSK. (a) Quercetin to CCND1; (b) quercetin to CXCL8; (c) tanshinone IIA to FOS; (d) tanshinone IIA to JUN; (e) naringenin to MAPK3; (f) quercetin to MMP9; (g) quercetin to MYC; (h) quercetin to PTGS2; (i) tanshinone IIA to TP53; (j) luteolin to VEGFA.

the components of the Chinese herbs in JSK and their SCIrelated target genes may not be known, and we may have sampled only a portion of them. Both in vitro and in vivo experiments are needed to further validate our results. With the development of new analytical techniques, we anticipate that more compounds from Chinese herbs and additional therapeutic targets for SCI will be discovered and validated.

\section{Conclusion}

Based on a combination of network pharmacology approach, gene expression validation, and molecular docking, we uncovered potential genes, proteins, and molecular pathways potentially regulated by JSK compounds for the treatment of SCI. Key bioactive compounds of JSK against SCI include quercetin, luteolin, kaempferol, tanshinone IIA, 7-O-methylisomucronulatol, and nobiletin. Ten genes were identified as important therapeutic SCI-related target genes of JSK, including CCND1, CXCL8, FOS, JUN, MAPK3, MMP9, MYC, PTGS2, TP53, and VEGFA. In addition, we demonstrated that JSK might provide therapeutic effects for SCI by regulating the pathways associated with reducing inflammatory response and regenerating nerve cells, including TNF, HIF-1, Toll-like receptor, and PI3K-Akt signaling pathways. This study provides additional insights into TCM therapies for treating SCI and lays a foundation for further experimental studies.

\section{Abbreviations}

AKT1: $\quad$ AKT serine/threonine kinase 1

CASP3: Caspase 3
CCND1: Cyclin D1

CXCL8: C-X-C motif chemokine ligand 8

EGF: Epidermal growth factor

EGFR: Dermal growth factor receptor

FOS: Proto-oncogene

GO: $\quad$ Gene ontology

IL6: Interleukin 6

JUN: Jun proto-oncogene

KEGG: Kyoto Encyclopedia of Genes and Genomes

MAPK1: Mitogen-activated protein kinase 1

MAPK3: Mitogen-activated protein kinase 3

MAPK8: Mitogen-activated protein kinase 8

MMP2: Matrix metallopeptidase 2

MMP9: Matrix metallopeptidase 9

MYC: $\quad$ MYC proto-oncogene

PPI: Protein-protein interaction

PTGS2: Prostaglandin-endoperoxide synthase 2

ROC: Receiver operating characteristic curve

SCI: $\quad$ Spinal cord injury

STAT3: Signal transducer and activator of transcription 3

TCM: Traditional Chinese medicine

TP53: $\quad$ Tumor protein $\mathrm{p} 53$

VEGFA: Vascular endothelial growth factor A.

\section{Data Availability}

The data used and/or analyzed during the current study are available from the corresponding author upon reasonable request.

\section{Conflicts of Interest}

The authors declare that they have no conflicts of interest regarding the publication of this paper. 


\section{Acknowledgments}

This study was supported by grants from the Science and Technology Development Program of TCM of Jiangsu Province (YB2020042) and the Wuxi Science and Technology Bureau Project (SKJJZD19). The authors greatly appreciated TopEdit (https://www.topeditsci.com) for the linguistic assistance during the preparation of this manuscript.

\section{References}

[1] G. Courtine and M. V. Sofroniew, "Spinal cord repair: advances in biology and technology," Nature Medicine, vol. 25, no. 6, pp. 898-908, 2019.

[2] NSCIS Center, "Spinal cord injury (SCI) 2016 facts and figures at a glance," The Journal of Spinal Cord Medicine, vol. 39, no. 4, pp. 493-494, 2016.

[3] M. G. Fehlings, L. A. Tetreault, J. R. Wilson et al., "A clinical practice guideline for the management of acute spinal cord injury: introduction, rationale, and scope," Global Spine Journal, vol. 7, no. 3, pp. 84s-94s, 2017.

[4] M. J. Eckert and M. J. Martin, "Trauma:Spinal cord Injury," Surgical Clinics of North America, vol. 97, no. 5, pp. 1031-1045, 2017.

[5] M. Karsy and G. Hawryluk, "Modern medical management of spinal cord injury," Current Neurology and Neuroscience Reports, vol. 19, no. 9, p. 65, 2019.

[6] Y. Lu, J. Yang, X. Wang et al., "Research progress in use of traditional Chinese medicine for treatment of spinal cord injury," Biomedicine \& Pharmacotherapy, vol. 127, Article ID 110136, 2020.

[7] J. H. Sun, F. Sun, B. Yan, J. Y. Li, and D. L. Xin, "Data mining and systematic pharmacology to reveal the mechanisms of traditional Chinese medicine in mycoplasma pneumoniae pneumonia treatment," Biomedicine and Pharmacotherapy, vol. 125, Article ID 109900, 2020.

[8] J.-W. Wang, M. Wu, and G.-C. Huang, "Effect of Jisuikang (脊髓康) on kinetic dysfunction in patients after spinal injury," Chinese Journal of Integrative Medicine, vol. 14, no. 3, pp. 190-193, 2008.

[9] M. Yong, W. Mao, and W. Jian-Wei, "28 cases of spinal cord injury treated with Jisuikang," Liaoning Journal of Traditional Chinese Medicine, vol. 4, p. 309, 2017.

[10] M. Yong, W. Mao, and W. Jian-Wei, "Jisuikang in the treatment of 19 cases of dysuria after spinal cord injury," Jiangsu Journal of Traditional Chinese Medicine, vol. 4, p. 32, 2004.

[11] J. W. Wang, J. F. Yang, Y. Ma et al., "Nogo-A expression dynamically varies after spinal cord injury," Neural Regeneration Research, vol. 10, no. 2, pp. 225-229, 2015.

[12] W. L. You, Y. F. Zhang, J. F. Chen, H. Yin, J. F. Yang, and J. W. Wang, "Effects of Jisuikang on Nogo-NgR gene expression in spinal cord rats with injury," China Journal Of Orthopaedics And Traumatology, vol. 28, no. 3, pp. 235-239, 2015.

[13] Y. Guo, Y. Ma, Y. L. Pan, S. Y. Zheng, J. W. Wang, and G. C. Huang, "Jisuikang, a Chinese herbal formula, increases neurotrophic factor expression and promotes the recovery of neurological function after spinal cord injury," Neural Regeneration Research, vol. 12, no. 9, pp. 1519-1528, 2017.

[14] C. Wu, Y. Zhou, P. Tu et al., "Jisuikang promotes the repair of spinal cord injury in rats by regulating NgR/RhoA/ROCK signal pathway," Evidence-Based Complementary and Alternative Medicine, vol. 2020, Article ID 9542359, 13 pages, 2020.
[15] A. L. Liu and G. H. Du, "Network pharmacology:New guidelines for drug discovery," Acta Pharmaceutica Sinica, vol. 45, no. 12, pp. 1472-1477, 2010.

[16] L. Gao, X.-D. Wang, Y.-Y. Niu et al., "Molecular targets of Chinese herbs: a clinical study of hepatoma based on network pharmacology," Scientific Reports, vol. 6, no. 1, Article ID 24944, 2016.

[17] H. Lin, X. Wang, L. Wang et al., "Identified the synergistic mechanism of drynariae rhizoma for treating fracture based on network pharmacology," Evidence-Based Complementary and Alternative Medicine, vol. 2019, Article ID 7, 19 pages, 2019.

[18] A. L. Hopkins, "Network pharmacology: the next paradigm in drug discovery," Nature Chemical Biology, vol. 4, no. 11, pp. 682-690, 2008.

[19] H. Zhao, Y. Shan, Z. Ma, M. Yu, and B. Gong, "A network pharmacology approach to explore active compounds and pharmacological mechanisms of epimedium for treatment of premature ovarian insufficiency," Drug Design, Development and Therapy, vol. 13, pp. 2997-3007, 2019.

[20] H. Lin, X. Wang, M. Liu et al., "Exploring the treatment of COVID-19 with yinqiao powder based on network pharmacology," Phytotherapy Research:PTR, vol. 35, 2021.

[21] H. Bao, H. Guo, Z. Feng, and X. Li, "Deciphering the underlying mechanism of Xianlinggubao capsule against osteoporosis by network pharmacology," BMC Complementary Medicine and Therapies, vol. 20, no. 1, p. 208, 2020.

[22] J. Ru, P. Li, J. Wang et al., "TCMSP: a database of systems pharmacology for drug discovery from herbal medicines," Journal of Cheminformatics, vol. 6, no. 1, p. 13, 2014.

[23] Z. Liu, F. Guo, Y. Wang et al., "BATMAN-TCM: a bioinformatics analysis tool for molecular mechanism of traditional Chinese medicine," Scientific Reports, vol. 6, no. 1, Article ID 211, 2016.

[24] H.-Y. Xu, Y.-Q. Zhang, Z.-M. Liu et al., "ETCM: an encyclopaedia of traditional Chinese medicine," Nucleic Acids Research, vol. 47, pp. D976-d982, 2019.

[25] K. Tsaioun, B. J. Blaauboer, and T. Hartung, "Evidence-based absorption, distribution, metabolism, excretion (ADME) and its interplay with alternative toxicity methods," Altex, vol. 33, no. 4, pp. 343-358, 2016.

[26] G. Stelzer, N. Rosen, I. Plaschkes et al., "The GeneCards suite: from gene data mining to disease genome sequence analyses," Current Protocols in Bioinformatics, vol. 54, pp. 1-33, 2016.

[27] A. P. Davis, T. C. Wiegers, J. Wiegers et al., "CTD anatomy: analyzing chemical-induced phenotypes and exposures from an anatomical perspective, with implications for environmental health studies," Current Research in Toxicology, vol. 2, pp. 128-139, 2021.

[28] H. Chen and P. C. Boutros, "VennDiagram: a package for the generation of highly-customizable Venn and Euler diagrams in R," BMC Bioinformatics, vol. 12, no. 1, p. 35, 2011.

[29] P. Shannon, A. Markiel, O. Ozier et al., "Cytoscape: a software environment for integrated models of biomolecular interaction networks," Genome Research, vol. 13, no. 11, pp. 2498-2504, 2003.

[30] D. W. Huang, B. T. Sherman, and R. A. Lempicki, "Bioinformatics enrichment tools: paths toward the comprehensive functional analysis of large gene lists," Nucleic Acids Research, vol. 37, no. 1, pp. 1-13, 2009.

[31] D. Szklarczyk, A. Franceschini, S. Wyder et al., "STRING v10: Protein-protein interaction networks, integrated over the tree of life," Nucleic Acids Research, vol. 43, pp. D447-D452, 2015.

[32] Y. Tang, M. Li, J. Wang, Y. Pan, and F.-X. Wu, "CytoNCA: a cytoscape plugin for centrality analysis and evaluation of 
protein interaction networks," Biosystems, vol. 127, pp. 67-72, 2015.

[33] P. W. Rose, A. Prlić, A. Altunkaya et al., "The RCSB protein data bank:integrative view of protein, gene and 3D structural information," Nucleic Acids Research, vol. 45, pp. D271-d281, 2017.

[34] O. Trott and A. J. Olson, "AutoDock Vina:Improving the speed and accuracy of docking with a new scoring function, efficient optimization, and multithreading," Journal of Computational Chemistry, vol. 31, no. 2, pp. 455-461, 2010.

[35] T. Xu, C. Ma, S. Fan et al., "Systematic understanding of the mechanism of baicalin against ischemic stroke through a network pharmacology approach," Evidence-Based Complementary and Alternative Medicine, vol. 2018, Article ID 2582843, 11 pages, 2018.

[36] P. Yang, A. Chen, Y. Qin et al., "Buyang huanwu decoction combined with BMSCs transplantation promotes recovery after spinal cord injury by rescuing axotomized red nucleus neurons," Journal of Ethnopharmacology, vol. 228, pp. 123-131, 2019.

[37] H. Xu, Y. Yang, Q.-W. Deng et al., "Governor vessel electroacupuncture promotes the intrinsic growth ability of spinal neurons through activating calcitonin gene-related peptide/ $\alpha$-calcium/calmodulin-dependent protein kinase/neurotrophin-3 pathway after spinal cord injury," Journal of Neurotrauma, vol. 38, no. 6, pp. 734-745, 2021.

[38] Y. Song, J. Liu, F. Zhang, J. Zhang, T. Shi, and Z. Zeng, "Antioxidant effect of quercetin against acute spinal cord injury in rats and its correlation with the p38MAPK/iNOS signaling pathway," Life Sciences, vol. 92, no. 24-26, pp. 1215-1221, 2013.

[39] J. Fu, H. Sun, Y. Zhang et al., "Neuroprotective effects of luteolin against spinal cord ischemia-reperfusion injury by attenuation of oxidative stress, inflammation, and apoptosis," Journal of Medicinal Food, vol. 21, no. 1, pp. 13-20, 2018.

[40] J. Fu, W. Xu, Y. Zhang, H. Sun, and J. Zhao, "Luteolin modulates the NF-E2-related factor 2/glutamate-cysteine ligase pathway in rats with spinal cord injury," Journal of Medicinal Food, vol. 24, no. 3, pp. 218-225, 2021.

[41] Z. Liu, X. Yao, B. Sun et al., "Pretreatment with kaempferol attenuates microglia-mediate neuroinflammation by inhibiting MAPKs-NF- $\kappa \mathrm{B}$ signaling pathway and pyroptosis after secondary spinal cord injury," Free Radical Biology and Medicine, vol. 168, pp. 142-154, 2021.

[42] Y. D. Yang, X. Yu, X. M. Wang, X. H. Mu, and F. He, "Tanshinone IIA improves functional recovery in spinal cord injury-induced lower urinary tract dysfunction," Neural Regeneration Research, vol. 12, no. 2, pp. 267-275, 2017.

[43] C. Wu, H. Xu, J. Li et al., "Baicalein attenuates pyroptosis and endoplasmic reticulum stress following spinal cord ischemiareperfusion injury via autophagy enhancement," Frontiers in Pharmacology, vol. 11, Article ID 1076, 2020.

[44] M. B. Orr and J. C. Gensel, "Spinal cord injury scarring and inflammation:therapies targeting glial and inflammatory responses," Neurotherapeutics, vol. 15, no. 3, pp. 541-553, 2018.

[45] H. Lu, L. H. Zhang, L. Yang, and P. F. Tang, "The PI3K/Akt/ FOXO3a pathway regulates regeneration following spinal cord injury in adult rats through TNF- $\alpha$ and p27kip1 expression," International Journal of Molecular Medicine, vol. 41, no. 5, pp. 2832-2838, 2018.

[46] X. Li, X. Lou, S. Xu, J. Du, and J. Wu, "Hypoxia inducible factor-1 (HIF-1 $\alpha$ ) reduced inflammation in spinal cord injury via miR-380-3p/ NLRP3 by Circ 0001723," Biological Research, vol. 53, no. 1, p. 35, 2020.
[47] W. Zhu, X. Chen, L. Ning, and K. Jin, "Network analysis reveals TNF as a major hub of reactive inflammation following spinal cord injury," Scientific Reports, vol. 9, no. 1, p. 928, 2019.

[48] Y. Wang, Q. J. Kong, J. C. Sun et al., "Protective effect of epigenetic silencing of cyclinD1 against spinal cord injury using bone marrow-derived mesenchymal stem cells in rats," Journal of Cellular Physiology, vol. 233, no. 7, pp. 5361-5369, 2018.

[49] T. Genovese, E. Esposito, E. Mazzon et al., "Evidence for the role of mitogen-activated protein kinase signaling pathways in the development of spinal cord injury," Journal of Pharmacology and Experimental Therapeutics, vol. 325, no. 1, pp. 100-114, 2008.

[50] N. Jing, B. Fang, Z. Li, and A. Tian, "Exogenous activation of cannabinoid-2 receptor modulates TLR4/MMP9 expression in a spinal cord ischemia reperfusion rat model," Journal of Neuroinflammation, vol. 17, no. 1, p. 101, 2020.

[51] Y. Joshi, M. G. Sória, G. Quadrato et al., "The MDM4/MDM2p53-IGF1 axis controls axonal regeneration, sprouting and functional recovery after CNS injury," Brain: A Journal of Neurology, vol. 138, pp. 1843-1862, 2015.

[52] N. Du, H. Li, C. Sun et al., "Adult astrocytes from reptiles are resistant to proinflammatory activation via sustaining Vav1 expression," Journal of Biological Chemistry, vol. 296, Article ID 100527, 2021.

[53] T. Zhang, G. Gao, and F. Chang, “miR-152 promotes spinal cord injury recovery via c-jun amino terminal kinase pathway," European Review for Medical and Pharmacological Sciences, vol. 23, no. 1, pp. 44-51, 2019.

[54] X. H. Yue, L. Guo, Z. Y. Wang, and T. H. Jia, "Inhibition of miR-17-5p promotes mesenchymal stem cells to repair spinal cord injury," European Review for Medical and Pharmacological Sciences, vol. 23, no. 9, pp. 3899-3907, 2019. 